\title{
EDITORIALE
}

\section{COME CONTRASTARE LA CATTIVA REPUTAZIONE DEL MARKETING}

\author{
Gianni Cozzi *
}

Vi sono oggi, a mio avviso, tre diverse correnti di opinione che convergono nel sottovalutare il ruolo del marketing come "filosofia" e come prassi fondamentale per fronteggiare la crisi economica e per percorrere vie d'uscita dalla crisi meno incerte per le imprese e più coerenti con le mutate aspettative dei loro clienti e della collettività.

La prima serpeggia all'interno dei quartieri alti di alcune imprese, che considerano il marketing come se si trattasse di un centro di costi, indispensabili per conservare e, se possibile, per migliorare il loro posizionamento competitivo, ma non espandibili, anzi comprimibili in periodi di "vacche magre".

La seconda, alquanto sorprendente, serpeggia anch'essa all'interno di alcune imprese ed anche nel variegato universo degli studiosi e dei professionals del marketing e della comunicazione. Per interpretare questa corrente di opinione sarebbe necessario avvalersi, anzitutto, del contributo degli psicologi. C'è infatti, tra gli addetti ai lavori, chi quasi "si vergogna" di fare marketing, chi si sente a suo agio solo percorrendo vie nuove ed incerte di reverse marketing, che, seppure da un lato quasi imposte, in qualche caso, dall'individualizzazione delle aspettative e dall'aumento del potere contrattuale dei clienti, dall'altro facilitate dall'evoluzione delle tecnologie, non possono comunque essere fini a se stesse; c'è infine chi ritiene opportuno rinchiudersi in campi sempre più specialistici di analisi, anch'essi necessari, non preoccupandosi però dell'uso che può essere fatto dei loro risultati.

Nei confronti di queste due correnti di opinione ben ha fatto Enrico Valdani, nel suo breve e molto efficace editoriale pubblicato sul numero prece-

\footnotetext{
* Professore ordinario della Facoltà di Economia dell’Università di Genova.
} 
dente della nostra rivista, sia a richiamare i fraintendimenti concettuali di fondo ad esse sottostanti, sia ad indicare le componenti della "bussola del marketing", tra loro strettamente connesse, necessarie per orientare e correggere la rotta delle imprese (e di altre organizzazioni), specie nell'attuale situazione di difficoltà e di incertezza, evidenziando anche il problema dell'ancora insoddisfacente allineamento tra le metriche specialistiche del marketing e quelle del business management.

La terza corrente di opinione, spesso ipercritica nei confronti del marketing in quanto tale, non solo di alcune sue manifestazioni percepite come distorte o distorsive non è composta da "addetti ai lavori" ma da persone esterne che del marketing hanno una visione in genere molto approssimativa e, al tempo stesso, dicotomica.

Da un lato, infatti, riconoscono che il marketing, se attuato correttamente, dovrebbe spingere le imprese a considerare la maggiore soddisfazione possibile dei loro clienti come il loro scopo primario, in assenza della quale, specie in un periodo di crisi, potrebbe essere messa in discussione la loro stessa capacità di sopravvivenza. Dall'altro, osservando le manifestazioni più esteriori dei processi di marketing, in modo particolare i contenuti e l'intensità della comunicazione commerciale attraverso i mass media, e non compiendo alcuno sforzo per cercare di "mettersi nei panni" degli individui, molto diversi da loro, che compongono il target cui sono diretti tali messaggi, si sentono continuamente "traditi" dal marketing.

Si tratta, nella maggioranza dei casi, di persone serie che, sul piano cognitivo e degli atteggiamenti, nonché, almeno in parte, dei loro concreti comportamenti, non si limitano a stigmatizzare gli alti e, a loro avviso, ingiustificati livelli di prezzo che riescono a "spuntare" le marche più affermate specie nel campo dei beni di consumo immediato, ma che cercano anche di esprimere la loro contrarietà nei confronti di tutto ciò che può contribuire, anche indirettamente, a processi di sviluppo non sostenibili sotto il profilo ambientale, economico e sociale.

La loro influenza sull'opinione pubblica complessiva sta decisamente aumentando, in una situazione nella quale, anche a motivo di una continua riflessione collettiva sulle determinanti non solo economico-finanziarie, ma anche socio-culturali della crisi, i valori su cui si regge la sostenibilità dello sviluppo tendono a diffondersi rapidamente.

In sostanza, i messaggi di questa corrente di opinione che possono notevolmente influire in modo negativo sulla reputazione del marketing sono di due tipi.

In primo luogo il marketing starebbe facendo troppo poco per predisporre un'uscita dalla crisi che non riproponga le contraddizioni che hanno contribuito ad innescarla. In secondo luogo i valori che il marketing continua a fare propri e ad amplificare sarebbero in molti casi l'esatto opposto rispetto a quelli da assumere come punti di riferimento per cercare di per- 
correre una strada di sviluppo sostenibile, ammesso che vi sia ancora il tempo per poterlo fare.

Rispondere a questa corrente di opinione in modo esclusivamente dialettico è - a mio avviso - sostanzialmente inutile, se non controproducente.

Ad esempio non è affatto sufficiente illustrare casistiche di marketing (ve ne sono molte più di quante si è soliti supporre) che assumono valori coerenti con lo sviluppo sostenibile e che si avvalgono di politiche di mercato (specie di politiche innovative del prodotto) positive da questo punto di vista. Limitandosi a ciò, si finirebbe con l'accentuare la contrapposizione tra "buono" e "cattivo" marketing, assumendo di fatto la distinzione un po' manichea dei nostri interlocutori in modo acritico e quindi radicandola ulteriormente.

Può anche essere controproducente, perché da loro interpretabile come una prova di cinico relativismo, osservare che qualsiasi azione di marketing, per essere efficace, deve contribuire a creare e trasferire valore (non solo economico) ai suoi specifici clienti. Pertanto i valori ed i criteri di valutazione prevalenti in quello che un tempo veniva denominato il suo target sono il suo punto di innesco ed anche il momento finale della verità circa la sua appropriatezza. Non è, quindi, corretto giudicare un'azione di marketing dall'esterno, prescindendo dai riferimenti valoriali specifici del segmento di mercato e, al limite, della personalità individuale cui essa è diretta e pretendendo di poter generalizzare i propri riferimenti valoriali.

A mio avviso, per cercare di contrastare efficacemente la corrente di opinione qui considerata, è necessaria, da parte di chi cerca correttamente di fare marketing senza vergognarsene, una riflessione più pacata, non priva anche di alcuni spunti autocritici.

Occorre chiedersi, in primo luogo, se si è compiuto tutto il possibile, tenendo conto degli avanzamenti recenti degli studi di marketing e delle strumentazioni tecniche oggi disponibili, per intercettare effettivamente, anche in modo controintuitivo ed anche interpretando i segnali deboli di cambiamento e, in alcuni casi, coinvolgendo la creatività dei singoli clienti e delle loro comunità reali e virtuali, per individuare ciò che potrebbe costituire per i propri clienti un valore addizionale, coerente con una maggiore sostenibilità ambientale, economica e sociale, valore addizionale che, in loro, è spesso ancora allo stato latente, ma che è possibile cercare di concretizzare, rendere percepibile e trasmettere potenziando le proprie capacità distintive, specie di carattere innovativo.

In secondo luogo è necessario considerare la crescente importanza del marketing come strumento atto ad individuare il valore d'uso (non solo individuale, ma anche collettivo) degli attuali potenziali tecnologici, che spesso derivano dall'integrazione di diverse discipline scientifiche, anche al fine di svilupparne applicazioni ed utilizzazioni innovative coerenti con uno sviluppo sostenibile. 
In terzo luogo occorre riflettere sul ruolo di alcune istituzioni pubbliche, espressione di soggettività collettive emergenti, nel porre condizioni coerenti con uno sviluppo sostenibile, producendo norme che possono tradursi in sistemi di vincoli o di incentivi, oppure intervenendo nell'orientare o organizzare sistemi esterni rilevanti per il proprio settore di attività (ad esempio un sistema energetico non più dipendente in misura preponderante da fonti fossili nella generazione di elettricità rilevante per il settore auto in una prospettiva di diffusione della propulsione completamente elettrica senza emissioni di anidride carbonica).

Nella logica tradizionale del marketing le decisioni normative e gli indirizzi delle istituzioni pubbliche venivano considerati come elementi dell'ambiente generale suscettibili di analisi solo ed in quanto potenziali generatori di opportunità o di minacce.

Questo approccio probabilmente va oggi superato, assumendo una più decisa prospettiva di carattere relazionale-interattivo, nel convincimento che gran parte dei processi di sviluppo sostenibile possibili coinvolgono, come attori primari dal lato dell'offerta, le imprese ed alcune istituzioni pubbliche, ossia soggetti necessariamente portatori di finalità caratteristiche diverse. A mio avviso la capacità degli studiosi e degli operatori di marketing di cogliere questa nuova prospettiva di interazione, senza scadere - come è spesso avvenuto in passato ed avviene anche oggi- in comportamenti opportunistici, è una sfida non secondaria che si pone al marketing (e non solo) e che può influire non poco anche sulla sua reputazione.

Da ultimo, ma non meno importante, non si può perdere di vista che la corrente di opinione considerata in questo intervento, pur essendo ipercritica nei confronti del marketing, non nega che un "buon" marketing dovrebbe anzitutto creare valore e trasferirlo al cliente. Essa si sente spesso "tradita" dal marketing proprio perché percepisce che una parte consistente del valore creato non viene poi di fatto trasferito, ma viene trattenuto dall'impresa che compie l'azione di marketing.

Questa percezione la porta a scoprire, ad ogni piè sospinto, una verità che, per gli studiosi e per gli operatori di marketing, è quasi ovvia, caricandola di connotazioni negative spesso esasperate. Il marketing, infatti, è una sorta di "Giano bifronte", in quanto da un lato privilegia la creazione ed il trasferimento di valore per il cliente come condizione che giustifica la stessa esistenza delle imprese, dall'altro sviluppa politiche di mercato finalizzate a conservare ed accrescere il valore trattenuto dall'impresa anche a scapito di quello concretamente trasferito al cliente.

Di fronte a questa "scoperta", tutt'altro che nuova, a mio avviso è essenziale, con onestà, evitare di accreditare versioni edulcorate o ideologiche dei principi e della prassi del marketing manageriale. In particolare non mi sembra affatto rilevante, da questo punto di vista, osservare che sono mutati i rapporti tra le imprese ed i loro clienti, un tempo ispirati a lo- 
giche unidirezionali e dirigistiche, oggi sempre più tendenti a relazioni bidirezionali di carattere dialogico. Tutto questo è vero e bisogna tenerne conto se si esaminano i processi di creazione del valore, che oggi coinvolgono in maggiore misura il cliente, anche a livello individuale, la sua discrezionalità e la sua creatività. Tuttavia il problema della distribuzione del valore creato, nel marketing business-to-consumers, non cambia in modo sostanziale.

L'articolazione dei principi-base del marketing management, la loro estensione a qualsiasi relazione di scambio anche di carattere extra economico, il riconoscimento di una condizione di reciprocità, in uno scambio rispondente alle logiche del marketing, tra i vantaggi conseguibili dalle parti coinvolte, non hanno infatti dissipato l'ambiguità di un paradigma che continua, seppure tra le righe, a presentare il marketing come una sorta di "rivoluzione copernicana" in grado di modificare anche la finalità caratteristica delle imprese.

Basta considerare le teorie riguardanti la creazione ed il trasferimento del valore, oggi in voga, per rendersi conto del permanere di questa ambiguità originaria. Molto raramente, infatti, si distinguono, in queste teorie, in modo sufficientemente netto, le diverse determinanti del valore-costo, del valore di scambio e del valore d'uso, dando per scontato che il marketing, finalizzato comunque a creare, elevare e trasferire valore d'uso (per il cliente) non modifichi anche i meccanismi di formazione del valore di scambio, che - come è ben noto - dipendono dalle forme di mercato e, al loro interno, dagli stati della concorrenza e dalla sua regolazione.

Banalizzando al massimo il concetto testè richiamato, la soddisfazione del singolo cliente corrisponde, in ultima analisi, alla differenza, da questi percepita, tra valore d'uso (comprensivo delle sue componenti immateriali) e valore di scambio, mentre la soddisfazione dell'impresa corrisponde, in ultima analisi, alla differenza tra valore di scambio e valore-costo. La finalità propria dell'impresa, in condizioni di razionalità limitata e di incertezza, non può che essere quella di realizzare una redditività sul capitale investito ritenuta soddisfacente, almeno nel medio periodo, da chi la governa in condizioni di rischio ritenute, anche nel breve periodo, fronteggiabili. In funzione di questa finalità per l'impresa è essenziale cercare di conservare e, se possibile, aumentare la differenza tra valore costo e valore di scambio dei suoi output, anche erodendo - se il mercato glielo consente - la differenza tra il loro valore d'uso ed il loro valore di scambio, peraltro spesso contestualmente aumentata dalle attività di marketing ed utilizzando, a tal fine, strategie e politiche di mercato che, se necessario, introducono e potenziano, almeno pro-tempore, stati della concorrenza meno aperti e meno dinamici.

Con altre parole, l'aumento della soddisfazione del cliente, obiettivo innegabile del marketing, non modifica la finalità caratteristica dell'impresa, ma è ad essa strumentale. 


\section{G. Cozzi}

Inoltre, in una corretta logica di marketing, alla sovranità di ogni consumatore (intesa come libertà di scelta tra le alternative disponibili, entro i propri vincoli di bilancio, ed anche di proposta di nuove alternative) si accompagna comunque la sovranità di ogni impresa (intesa come libertà di scelta, tra le alternative da essa ritenute concretamente configurabili e fattibili, di quelle coerenti con le sue capacità distintive, ritenute più convenienti e meno rischiose). Sulle valutazioni attraverso le quali si estrinseca la sovranità dell'impresa, quelle rispondenti a logiche competitor oriented si integrano necessariamente con quelle customer oriented, specie nell'area delle decisioni di marketing.

Non è quindi corretto, prescindendo da una stringente valutazione delle forme di mercato e degli stati, anche temporanei, della concorrenza al loro interno, accreditare versioni ideologiche del paradigma di marketing, nelle quali si postula sempre e comunque una perfetta convergenza tra interessi individuali e interessi collettivi, tra soddisfazione dei clienti e soddisfazione degli azionisti (oggi sarebbe forse meglio parlare di soddisfazione del management), fino ad arrivare, in alcuni casi, a prospettare il marketing come un elemento che può potenziare il ruolo della "mano invisibile" del mercato di smithiana memoria, dimenticandosi che, dal punto di vista del marketing, è per definizione irrilevante - come modello teorico di riferimento - la forma di mercato di concorrenza perfetta. Queste versioni vanno chiaramente denunciate, anzitutto dagli studiosi di marketing, perché, nonostante le loro apparenze, nuocciono profondamente non solo alla coerenza, ma anche alla reputazione della disciplina.

Purtroppo, invece, in molti manuali di marketing che vanno per la maggiore, dopo le prime venti-cinquanta pagine di stampo prevalentemente ideologico, con cui erroneamente i loro autori ritengono di accreditare la rilevanza sociale del marketing, seguono duecento-trecento pagine nelle quali essi illustrano dettagliatamente tutte le "astuzie" che il marketing può suggerire per conciliare (ovviamente a vantaggio dell'impresa), la creazione di valore per il cliente e l'incremento di valore per l'azionista (o, meglio, per il management).

Questo modo di procedere è fortemente diseducativo perché rivolto anche - e principalmente - agli studenti universitari, le cui capacità critiche sono ancora poco sviluppate, e non contribuisce in ogni caso a migliorare la reputazione della disciplina.

Nella mia ormai tarda età e seguendo la mia propensione a cercare di coltivare sia gli studi di economia e gestione delle imprese sia quelli di economia industriale, rimpiango quanto emergeva, molti anni or sono, nei dibattiti sul ruolo pro o anticompetitivo di alcuni rilevanti investimenti di marketing, dibattiti ai quali partecipavano spesso economisti industriali assai noti e apprezzati. Ricordo che il marketing era sottoposto frequentemente ad analisi critiche sui suoi effetti per l'economia collettiva, ma la 
sua reputazione non veniva compromessa perché il marketing era considerato come uno specchio fedele del sistema capitalistico di allora, sistema di cui venivano apertamente valutate le contraddizioni e le divergenze tra interessi collettivi ed interessi individuali che lo caratterizzavano ma di cui non veniva perso di vista - come sottolineava esplicitamente Kaldor (1950) - l'innegabile e continuo apporto complessivo non solo alla crescita, ma anche allo sviluppo economico (ricordo, ad esempio, i contributi di: Bain (1956); Sylos Labini (1956); Joan Robinson (1963)).

Con il successo dell'operazione cultuale tendente non solo (e non tanto) ad accreditare la cosiddetta "rivoluzione copernicana" del marketing management, ma anche (e specialmente) a porre ai margini delle teorie economiche dominanti le voci critiche meno allineate con il "pensiero unico" sulle "magnifiche sorti e progressive" del capitalismo industriale e finanziario nella sua versione statunitense, nonché dei suoi principali strumenti, i dibattiti di allora sono rimasti poco più che una reminiscenza storica. Penso, invece, che varrebbe la pena di riprenderne alcuni spunti di riflessione, nonostante le profonde differenze tra la realtà di allora e quella attuale, se si vuole cercare di migliorare la reputazione del marketing senza ricorrere ad una controproducente retorica o ad incerte "fughe in avanti".

La reputazione di un'area di studi e di un rilevante insieme di attività economiche ha infatti molto da guadagnare se ne vengono illustrate le reali finalità e le effettive connotazioni, con tutte le loro luci, ma anche con tutte le loro ombre. In effetti, però, da parte delle più qualificate rappresentanze degli studiosi e degli operatori di Marketing, a cominciare dall'American Marketing Association, ciò non avviene o si manifesta molto raramente. Come Società Italiana di Marketing, vogliamo seguire il loro esempio? 